\title{
Phase I Clinical Study of ZYAN1, A Novel Prolyl-Hydroxylase (PHD) Inhibitor to Evaluate the Safety, Tolerability, and Pharmacokinetics Following Oral Administration in Healthy Volunteers
}

\author{
Kevinkumar A. Kansagra ${ }^{1}$ - Deven Parmar ${ }^{1}$ Rajendra H. Jani ${ }^{1}$. \\ Nuggehally R. Srinivas ${ }^{1} \cdot$ Jason Lickliter $^{2} \cdot$ Harilal V. Patel $^{1}$ • \\ Devang P. Parikh ${ }^{1} \cdot$ Heather Heading ${ }^{3} \cdot$ Hardik B. Patel $^{1} \cdot$ Rahul J. Gupta $^{1}$. \\ Chintan Y. Shah ${ }^{1} \cdot$ Maulik R. Patel $^{1} \cdot$ Vyom N. Dholakia $^{4} \cdot$ Raghav Sukhadiya $^{4} \cdot$ \\ Mukul R. Jain ${ }^{1} \cdot$ Krupi V. Parmar ${ }^{1} \cdot$ Kinjal Barot $^{1}$ \\ Published online: 16 May 2017 \\ (C) The Author(s) 2017. This article is published with open access at Springerlink.com
}

\begin{abstract}
Objective This phase I study of ZYAN1 was conducted to evaluate the safety, tolerability, and pharmacokinetics following oral administration in healthy volunteers.

Methods The study was a randomized, double-blind, placebo-controlled phase I study carried out in two parts in addition to a third part involving an open-label study to evaluate the food/sex effect. A total of 100 subjects were enrolled into the study as follows: part I-single-dose study with ZYAN1 10, 25, 50, 100, 150, 200, and $300 \mathrm{mg}$ $(n=56)$; part II-multiple-dose study with every other day dosing of ZYAN1 100, 150, 200, and $300 \mathrm{mg}$ $(n=32)$; and part III-sex and food effect study with ZYAN1 $150 \mathrm{mg}$ ( $n=12$; open-label).

Results ZYAN1 was well-tolerated after single and multiple oral ascending doses. No drug-related serious adverse events were reported. Following a single ascending dose of ZYAN1, the maximum concentration $\left(C_{\max }\right)$ ranged from $566.47 \pm 163.03$ to $17,858.33 \pm 2899.19 \mathrm{ng} / \mathrm{mL}$ and the
\end{abstract}

Deven Parmar

deven.parmar@zyduscadila.com

1 Zydus Research Center, Survey No. 396/403, Sarkhej-Bavla N.H. No. 8A, Moraiya, Ahmedabad, Gujarat 382213, India

2 Division of Nucleus Network, Centre for Clinical Studies (CCS), Melbourne, Australia

3 CPR Pharma Services Pty Ltd, Thebarton, SA 5031, Australia median time to $C_{\max }\left(t_{\max }\right)$ was approximately $2.5 \mathrm{~h}$ for the studied 30-fold oral doses of ZYAN1. Regardless of single or multiple doses, mean $C_{\max }$ and area under the concentration-time curve from time zero to time $t\left(\mathrm{AUC}_{t}\right)$ values generally showed a dose-proportional increase. The mean elimination half-life $\left(t_{1 / 2}\right)$ of ZYAN1 ranged from 6.9 to $13 \mathrm{~h}$ with negligible accumulation. Following a single dose of ZYAN1, the mean serum erythropoietin (EPO) $C_{\max }$ values showed dose response (i.e., 6.6 and $79.9 \mathrm{mIU} / \mathrm{L}$ for 10 and $300 \mathrm{mg}$ ZYAN1 doses, respectively), while the time to mean maximal serum EPO concentrations ranged from 10 to $72 \mathrm{~h}$.

Conclusion Oral single (10-300 mg) and multiple dosing (100-300 mg) of ZYAN1 in healthy subjects was found to be safe and well-tolerated. With increasing ZYAN1 dose, there was almost a proportional increase in mean $C_{\max }$ and $\mathrm{AUC}_{t}$. The mean serum EPO concentrations showed a trend of dose response. Based on the $t_{1 / 2}$, pharmacodynamic activity, and lack of drug accumulation, a once every 2 days dosing regimen of ZYAN1 was appropriate for phase II study.

Trial Registration: Australian New Zealand Clinical Trials Registry trial ID ACTRN12614001240639. 


\section{Key Points}

A comprehensive phase I study evaluation of ZYAN1, a novel oral prolyl-hydroxylase (PHD) inhibitor for the management of anemia in chronic kidney diseases, was carried out in healthy volunteers.

ZYAN1 was safe and well-tolerated in healthy volunteers following single escalating oral doses (10-300 mg) and multiple (given once every 2 days) escalating oral doses (100-300 mg).

Without regard to single or multiple oral dose administration, both the mean maximum concentration $\left(C_{\max }\right)$ and area under the concentration-time curve from time zero to time $t\left(\mathrm{AUC}_{t}\right)$ of ZYAN1 generally increased in a ratio comparable to the dose increment ratio. Because the mean elimination half-life of ZYAN1 ranged from 6.9 to $13 \mathrm{~h}, \mathrm{ZYAN} 1$ was found to have negligible accumulation.

The measurement of serum erythropoietin (EPO) levels in healthy volunteers confirmed the pharmacodynamic effect as EPO increased with increasing ZYAN1 doses in relation to placebo.

On the basis of phase I data, ZYAN1 is recommended for phase II evaluation in a dose range of 100-200 mg every 2 days.

\section{Introduction}

The causes of anemia may include impaired red blood cell (RBC) production, increased RBC destruction, blood loss, and fluid overload [1,2]. Anemia can also be caused by a range of serious medical conditions such as chemotherapyinduced anemia (CIA), chronic kidney disease (CKD), and anemia of chronic disease (ACD) [3-5]. Currently available treatments of anemia typically rely on nutritional supplements, treatment of any underlying disorder, and administration of either RBCs or erythropoiesis-stimulating agents (ESAs) [6, 7].

In healthy non-anemic subjects, there is a dynamic, multi-faceted continuous process of erythropoiesis in place to meet the oxygen demands for the normal functioning of the body. Hypoxia-inducible factor (HIF) coordinates this process by mediating genes involved in erythropoiesis [8]. Erythropoietin (EPO) and iron are crucial to proper formation of RBCs. HIF promotes EPO secretion from the kidney and other non-renal sources (e.g., liver) and also upregulates the EPO receptor in the bone marrow. HIF also activates factors that improve iron absorption from the gut (e.g., transferrin, transferrin receptor, ferroprotein, ceruloplasmin, and divalent metal transporter), mobilization from storage sites, and transport to the bone marrow [9].

It has been observed that erythropoiesis is often suppressed in chronic inflammatory diseases [10]. Moreover, the failing kidneys in CKDs produce inadequate amounts of EPO, resulting in renal anemia [11]. This may be due to expression of hepcidin, an iron regulatory hormone that limits the availability of iron for erythropoiesis. Such functional iron deficiency is a common cause of suboptimal hemoglobin response to EPO analog therapy, and supplemental iron is commonly provided as a necessary adjuvant treatment to EPO analogs. HIF increases renal EPO production, thereby indirectly suppressing hepcidin. Hepcidin suppression in the liver permits increased ferroportin surface expression, which in turn increases iron availability for erythropoiesis [12].

Currently available therapies for anemia have certain safety issues, e.g., risk of iron overload with iron supplements, risk of hypertension, and adverse cardiovascular outcomes with ESAs, etc. [13]. Novel therapeutic strategies are required to replace these therapies and it is believed that modulation of the HIF pathway holds promise for this [14]. Encouraging results by targeting this pathway have already been observed from clinical trials supporting the possibility of pursuing an orally bioavailable therapy that is significantly less expensive than current treatments and thus generally more accessible for certain patient populations [15].

HIF-1 and HIF-2 likely regulate different sets of genes, although their downstream genes largely overlap. HIF-1 $\alpha$ is expressed under normoxic baseline conditions while $H I F-2 \alpha$ appears to be a key element in the hypoxic response. It is also observed that in certain situations, HIF$l \alpha$ controls the early response to hypoxia. HIF- $1 \alpha$ plays an important role in the cell-cycle regulation of hematopoietic stem cells. This effect is independent of EPO [16-18]. HIF1 is a heterodimer, consisting of an $\alpha$ (the regulatory subunit) and a $\beta$ subunit [19]. While the presence of the $\beta$ subunit is generally constant and in excess, the activity of the $\alpha$ subunit within the cell shows an oxygen-sensitive pattern (i.e., inversely related to the oxygen concentration) [20]. This orchestrating mode has been found to be mainly regulated by a family of prolyl-hydroxylase (PHD) enzymes, namely PHD1, PHD2, and PHD3 [21].

The PHD enzymes are critical in regulation of the HIF pathway as they hydroxylate HIF, leading to its ubiquitination and subsequent hydrolysis and destruction. Therefore, the inhibitors of HIF-PHD have the potential to mimic hypoxia, which can be useful for inducing erythropoiesis for the treatment of a variety of diseases such as anemia 
and hypoxia-related disorders (e.g., ulcerative colitis, myocardial ischemia, stroke, and metabolic disorders) [22, 23].

Considering the therapeutic potential of PHD inhibitors to address the unmet need for anemia, ZYAN1, a novel orally bioavailable drug, is being developed at the Zydus Research Center (Ahmedabad, Gujarat, India). During in vitro analysis in HepG2 cells, ZYAN1 showed HIF stabilization by inhibiting the PHD enzyme (concentration of drug producing 50\% inhibition [ $\left.\mathrm{IC}_{50}\right]$ : $11.2 \mu \mathrm{mol} / \mathrm{L}$ ) [24]. In an acute pharmacodynamic animal model, ZYAN1 showed a dose-related increase in serum EPO levels. In a repeated dose model, ZYAN1 showed a robust increase in erythropoiesis, resulting in normalization of hemoglobin levels in nephrectomized rats and mice. The effective dose for inducing hematopoiesis was observed to be $15 \mathrm{mg} / \mathrm{kg}$, with an alternate dosing pattern for a week. The preclinical pharmacokinetic data indicated that ZYAN1 is well-absorbed and highly bioavailable after oral dosing across the species tested. ZYAN1 is well-distributed, and it is partially excreted as unchanged drug in the urine and bile and showed minimal potential for cytochrome P450 (CYP)mediated drug-drug interactions [24]. It was observed that in single-dose studies, ZYAN1 was tolerated up to $300 \mathrm{mg} / \mathrm{kg}$ in mice, up to $800 \mathrm{mg} / \mathrm{kg}$ in rats, and up to $75 \mathrm{mg} / \mathrm{kg}$ in beagle dogs by the oral route [24]. Based on 1 -month toxicity studies, $60 \mathrm{mg} / \mathrm{kg}$ in rats and $15 \mathrm{mg} / \mathrm{kg}$ in dogs was considered the maximum tolerated dose (MTD) and $5 \mathrm{mg} / \mathrm{kg}$ was considered the no observed adverse effect level (NOAEL) in the 28-day toxicity studies in dogs.

\section{Materials and Methods}

This was a randomized, double-blind, placebo-controlled, phase I clinical study to evaluate the safety, tolerability, and pharmacokinetics of ZYAN1 following oral administration in healthy volunteers. The study was conducted at the Centre for Clinical Studies (CCS), Melbourne, Victoria, Australia after getting approval by the ethics committee and was duly registered on Australian New Zealand Clinical Trials Registry with trial ID ACTRN12614001240639. The study was conducted as per the International Conference on Harmonisation-Good Clinical Practice (ICH-GCP) guidelines. All study participants provided written informed consent for their willful participation in the study.

The primary objective of the study was to determine safety and tolerability of ZYAN1, which included assessments of adverse events (AEs), clinical and laboratory parameters, and monitoring of electrocardiogram and vital signs. The secondary endpoints were to determine the pharmacokinetics and pharmacodynamics of ZYAN1 after single and multiple oral dose administration in healthy male and female volunteers. Also, exploratory evaluation of sex and food effects was carried out on ZYAN1 for pharmacokinetic parameters.

\subsection{Participants}

Some of the key eligibility criteria for the study participants are as follows.

Inclusion criteria:

- healthy males or females between 18 and 65 years of age;

- hemoglobin value $12.8-17.5 \mathrm{~g} / \mathrm{dL}$ for males and $11.3-15.9 \mathrm{~g} / \mathrm{dL}$ for females;

- body weight $>55 \mathrm{~kg}$ and body mass index (BMI) within the range of $18-30 \mathrm{~kg} / \mathrm{m}^{2}$; and

- normal health as determined by personal medical history, clinical examination, and laboratory examinations.

Exclusion criteria:

- history or presence of any systemic disorders or diseases;

- abnormality in clinical examination, electrocardiogram (ECG), or laboratory examination; and

- for female volunteers, history of pregnancy or lactation in the past 3 months, fertile females not protected against pregnancy by adequate long-term anti-fertility measures, and those taking hormonal contraceptives.

Healthy male and female volunteers who satisfied the inclusion and exclusion criteria were enrolled into the study.

\subsection{Methodology}

The study schematic comprising parts I, II, and III is given in Fig. 1. Briefly, the study involving the following:

1. Part I: single ascending-dose trial. Involved seven independent panels, each of which comprised eight subjects of whom six were given successive single escalating doses of ZYAN1 (10, 25, 50, 100, 150, 200, and $300 \mathrm{mg}$ ) and two were given placebo. The oral administration of various ZYAN1 doses and matching placebo was carried out under fasting conditions (i.e., overnight fast).

2. Part II: multiple ascending-dose trial. Involved four independent panels, each of which consisted of eight subjects of whom six were given multiple escalating doses of ZYAN1 (100, 150, 200, and $300 \mathrm{mg}$ ) and two were given placebo on an every 2 days regimen (i.e., alternate-day dosing on Days 1, 3, and 5). Oral 


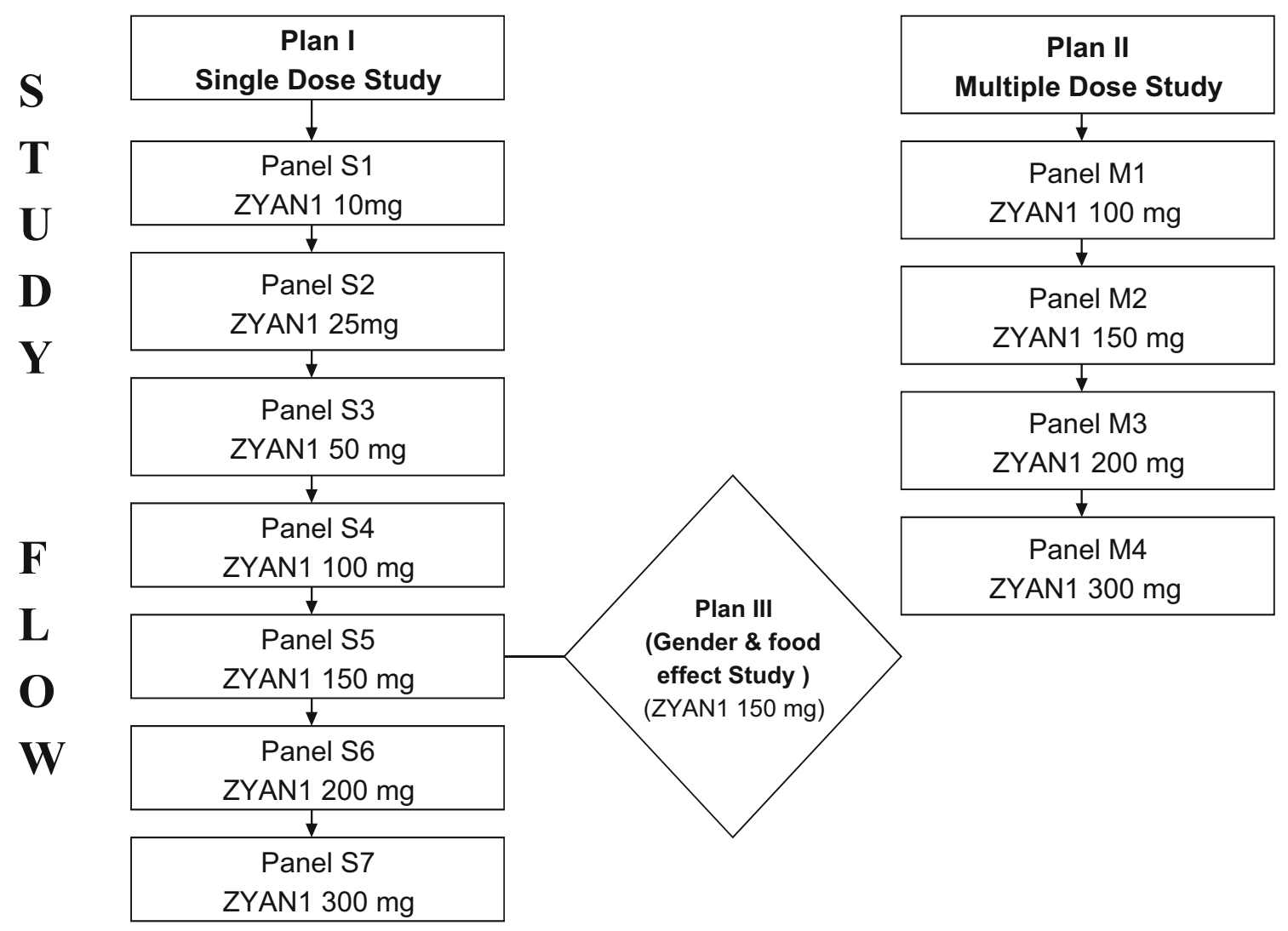

Fig. 1 Study flow

administration of various ZYAN1 doses and matching placebos was carried out under fasting conditions.

3. Part III: sex and food effect trial. Single-sequence, open-label, randomized, single-dose crossover study of two treatments (fasted vs. fed) of ZYAN1 with a washout period of at least 14 days between the two treatments. During period I, 12 subjects ( six men and six women) received a single $150 \mathrm{mg}$ oral dose of ZYAN1. During period II, ten subjects (five men and five women) received a high-fat and high-calorie (approximately 800-1000 calories) breakfast, which derives approximately 150, 250, and 500-600 calories from protein, carbohydrate, and fat, respectively, $30 \mathrm{~min}$ prior to the administration of a single $150 \mathrm{mg}$ oral dose of ZYAN1. In both periods, the participating subjects observed an overnight fast that lasted for at least $10 \mathrm{~h}$ prior to the oral dosing of ZYAN1 the next day.

\subsection{Safety Assessment}

All clinical signs and symptoms were monitored and frequent vital signs measurement, general and systemic clinical examination, 12-lead ECGs, as well as continuous ECG monitoring for $24 \mathrm{~h}$ post-dose (in parts I and II) were conducted to assess safety. Blood samples were collected to evaluate the safety of the standard battery of laboratory parameters after dosing. All AEs, including both observed and volunteered problems, complaints, signs, and symptoms were recorded irrespective of their association/causality with the investigational products and reported to the Institutional Review Board/Institutional Ethics Committee (IRB/IEC) of Centre for Clinical Studies (CCS), regulatory bodies, and the sponsor following respective guidelines. All AEs were coded using the current version of MedDRA ${ }^{\circledR}$ (Medical Dictionary for Regulatory Activities) (version 18.0) and concomitant medications were coded using the current version of the $W H O$ Drug Dictionary

\subsection{Blood Sample Collection}

For the single ascending-dose study (part I), samples for the pharmacokinetic assessment were collected before dosing, and at $0.5,1.0,1.5,2.0,2.5,3.0,3.5,4.0,5.0,6.0$, 8.0, 10.0, 12.0, 16.0, 24.0, 36.0, 48.0, and $72.0 \mathrm{~h}$ after the administration of the drug. For the multiple-dose study (part II), samples for the pharmacokinetic assessment were collected before dosing and at 0.5, 1.0, 1.5, 2.0, 2.5, 3.0, $3.5,4.0,5.0,6.0,8.0,10.0,12.0,16.0,24.0$, and $36.0 \mathrm{~h}$ after administration of Day 1 and Day 5 dosing. In addition, samples were collected before dosing and at 12.0, 
24.0 and $36.0 \mathrm{~h}$ after administration of Day 3 dosing and 48.0 and $72.0 \mathrm{~h}$ after administration of Day 5 dosing. Total blood loss for participation in the study was $217 \pm 5$, $452 \pm 5$, and $418 \pm 5 \mathrm{~mL}$ in parts I, II, and III, respectively.

\subsection{Urine Sample Collection}

For part I and III, urine samples were collected before dosing and then $0-12,12-24,24-36$, and $36-48 \mathrm{~h}$ following drug administration, while for part II, urine samples were collected before dosing, and then $0-12,12-24,24-36$, and $36-48 \mathrm{~h}$ after each dosing.

\section{Pharmacokinetic Blood Sampling}

In each study panel, a venous blood sample of $6 \mathrm{~mL}$ was collected for pre-dose and post-dose investigational medicinal product (IMP) in $\mathrm{K}_{2}$ EDTA vacutainers through a fresh venepuncture at various timepoints. The blood samples were placed in an ice bath, followed by dilution of the blood sample with equal volume of purified water (1:1) using a calibrated pipette. Samples were mixed well by vortex mixing and stored in freezer at $-80 \pm 10{ }^{\circ} \mathrm{C}$ until analysis.

Urine samples were collected at various timepoints in each study panel. The total volume of urine voided over each collection interval was collected in a single container and stored in a refrigerated condition. The total volume of urine collected was measured and recorded in the case report form. Aliquots of $5 \mathrm{~mL}$ of urine sample were prepared in pre-labelled tubes, and stored in a freezer at $-80 \pm 10{ }^{\circ} \mathrm{C}$ until analysis.

\subsection{Bioanalytical Procedures}

The estimation of ZYAN1 in diluted blood and urine was performed using a sensitive and specific liquid chromatography-tandem mass spectrometry (LC-MS/MS) method. The method was validated in accordance with the US Food and Drug Administration (FDA) Guidance for Bioanalytical Method Validation (May 2001)[25]. An analog internal standard (IOX2) was used for quantitative analysis of ZYAN1 in blood as well as in urine sample analysis. Chemically, IOX2 belongs to the quinolone chemotype class and has a glycinamide side chain, which are common between IOX2 and ZYAN1. IOX2 possess similar physicochemical properties to ZYAN1 and is highly suitable to use as an internal standard for mass spectrometry detection of ZYAN1. ZYAN1 and internal standard were separated on a C18 high-performance liquid chromatography (HPLC) column, and monitored by an API4000 MS/MS detector in positive MRM (multiple reaction monitoring) mode. The extraction of ZYAN1 and internal standard in acidified diluted blood samples and urine samples was carried out by protein precipitation using acetonitrile $(100 \%)$. The calibration standards were prepared in diluted blood and in urine, with a calibration curve (CC) range of $2-5000 \mathrm{ng} / \mathrm{mL}$ and a lower limit of quantitation of $2 \mathrm{ng} / \mathrm{mL}$. The quality control (QC) samples were analyzed intermittently with each batch of blood and urine samples at low, medium, and high levels and dilution QCs were within the acceptance limits of $\pm 15 \%$.

\subsection{Pharmacokinetic and Pharmacodynamic Assessments}

During all parts, blood samples were collected for the assessment of pharmacokinetic (maximum concentration [ $\left.C_{\max }\right]$, time to $C_{\max }\left[t_{\max }\right]$, area under the concentration-time curve [AUC] from time zero to time $t\left[\mathrm{AUC}_{t}\right]$, AUC from time zero to infinity [AUC $C_{\infty}$ ], elimination half-life $\left(t_{1 / 2}\right)$, elimination rate constant $[\lambda z]$, clearance $[C L]$, apparent volume of distribution $\left[V_{\mathrm{d}}\right]$, and accumulation index [for multiple-dose only]) and pharmacodynamic (hemoglobin, hematocrit, reticulocyte count, and EPO concentration) parameters.

\subsection{Statistical Methods}

Descriptive statistics were performed to assess pharmacokinetic, pharmacodynamic, and safety parameters. Pharmacodynamic parameters were evaluated using pretreatment and post-treatment clinical and laboratory findings. To determine whether there was a significant difference in the pharmacodynamic and safety variables post-dose compared with baseline, a paired $t$ test with a two-sided tail for an $\alpha=0.05$ significance level was performed. For the analysis of sex effect, data from fasted and fed subjects was used. To determine the effect of food in both male and female subjects, a $t$ test with a two-sided tail for an $\alpha=0.05$ significance level was performed. WinNonlin ${ }^{\circledR}$ Professional Software version 6.4 (Pharsight, Cary, NC, USA), SAS ${ }^{\circledR}$ version 9.4 (SAS Institute, Cary, NC, USA), and Microsoft Excel ${ }^{\circledR}$ (Microsoft Corp., Redmond, WA, USA) were used for pharmacokinetic analysis, randomization, and statistical analysis.

\subsection{Study Stopping Rule}

As this was a first trial of ZYAN1 in human subjects, for the safety of the participating subjects the protocol allowed for dose escalation or continued dosing within any dose group to be placed on hold if any of the following occurred:

- Hemoglobin rose more than $1 \mathrm{~g} / \mathrm{dL}$ at any point of time after dosing in four of six subjects in any panel. 
- A single serious AE (SAE) occurred in a volunteer on ZYAN1 during any panel that was possibly related to study drug; or two or more subjects experienced similar AEs of moderate severity in the same dosage group that were possibly related to the study drug; or pharmacokinetic saturation was reached or desired pharmacokinetic/pharmacodynamic effect was achieved.

\section{Results}

\subsection{Subjects' Disposition}

This phase I study was conducted from October 2014 to December 2015. Subjects were screened for inclusion and exclusion criteria and those found eligible were enrolled into the study after written informed consent was given by each of the participants.

A total of 100 subjects were enrolled into the study (56 in part I, 32 in part II, and 12 in part III) and included in the safety analysis. All of the subjects in part I $(n=56)$ and part II $(n=32)$ of the study who received a dose of the study drug (ZYAN1 or placebo) were included in the pharmacodynamic analysis. For part III, all subjects who received a dose of the study drug (ZYAN1) and completed the study as per protocol in each of period 1 and period 2 ( $n=10)$ were included in the pharmacodynamic analysis. In part III, ten of 12 subjects completed the study. One subject was withdrawn from the study due to non-compliance with the protocol and the other subject was withdrawn from the study due to an SAE of cellulitis that was not related to ZYAN1.

\subsection{Pharmacokinetic Results}

\subsubsection{Single Ascending-Dose Study}

The single-dose mean concentration versus time profiles of ZYAN1 for the various doses employed in the study are presented in Fig. 2. It is apparent from Fig. 3 that all of the plasma profiles were parallel across the absorptive, distributive, and elimination phases of ZYAN1. The mean pharmacokinetic data for ZYAN1 are depicted in Table 1. Regardless of the orally administered single-dose levels of ZYAN1, the $t_{\max }$ was relatively rapid (median $t_{\max }$ of approximately $2.5 \mathrm{~h}$ ). Both $C_{\max }$ and AUC parameters showed an almost dose-proportional increase over the studied 30-fold dose range of ZYAN1; there appeared to be a slight deviation from dose proportionality at higher oral doses of ZYAN1 (Fig. 3). The $t_{1 / 2}$ of ZYAN1 (range

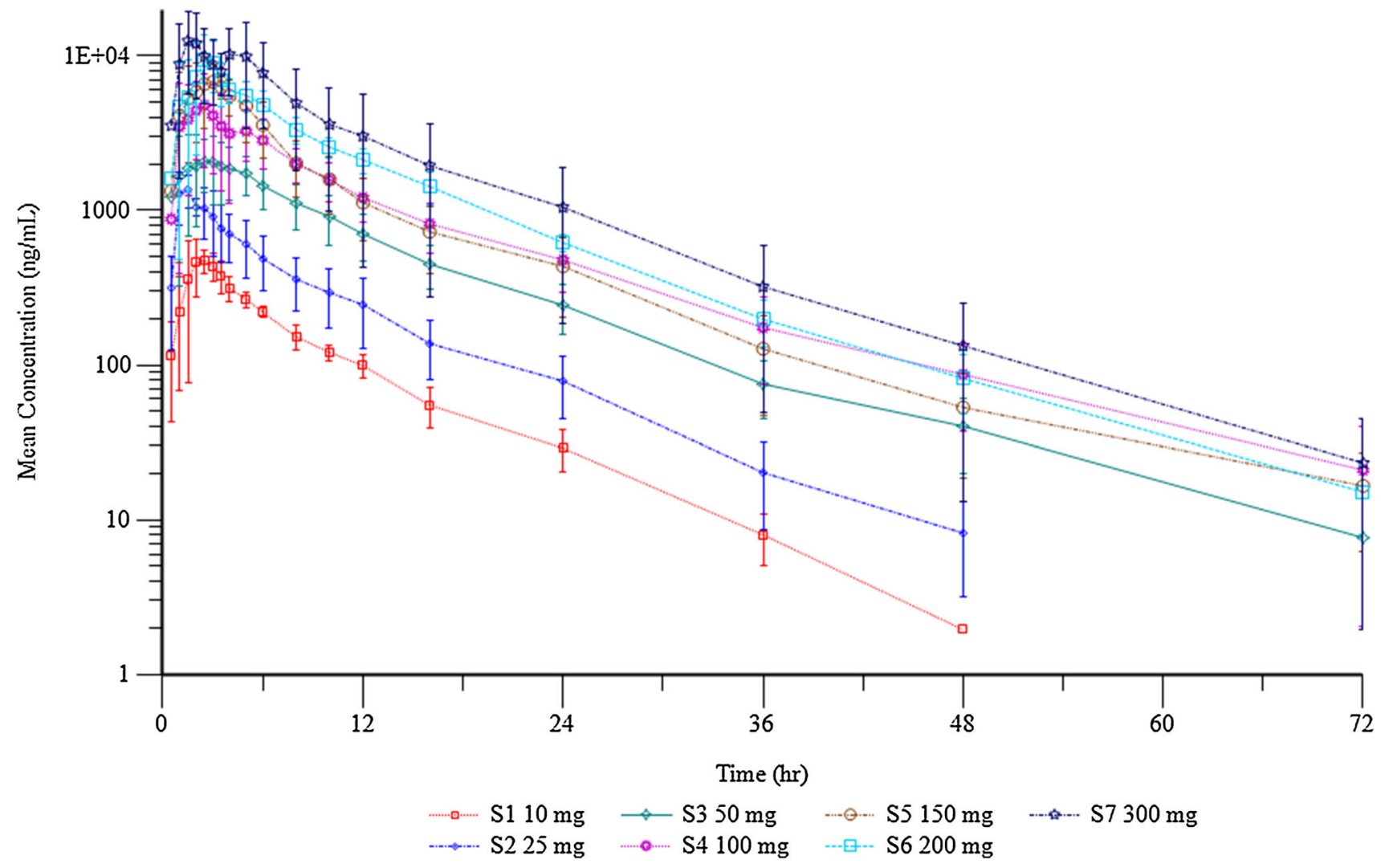

Fig. 2 Mean concentration vs. time graph with error bars for part I 
Fig. 3 Dose linearity plot of maximum concentration $\left(C_{\max }\right)$ and area under the concentration-time curve from time zero to time $t\left(\mathrm{AUC}_{t}\right)$ for part I

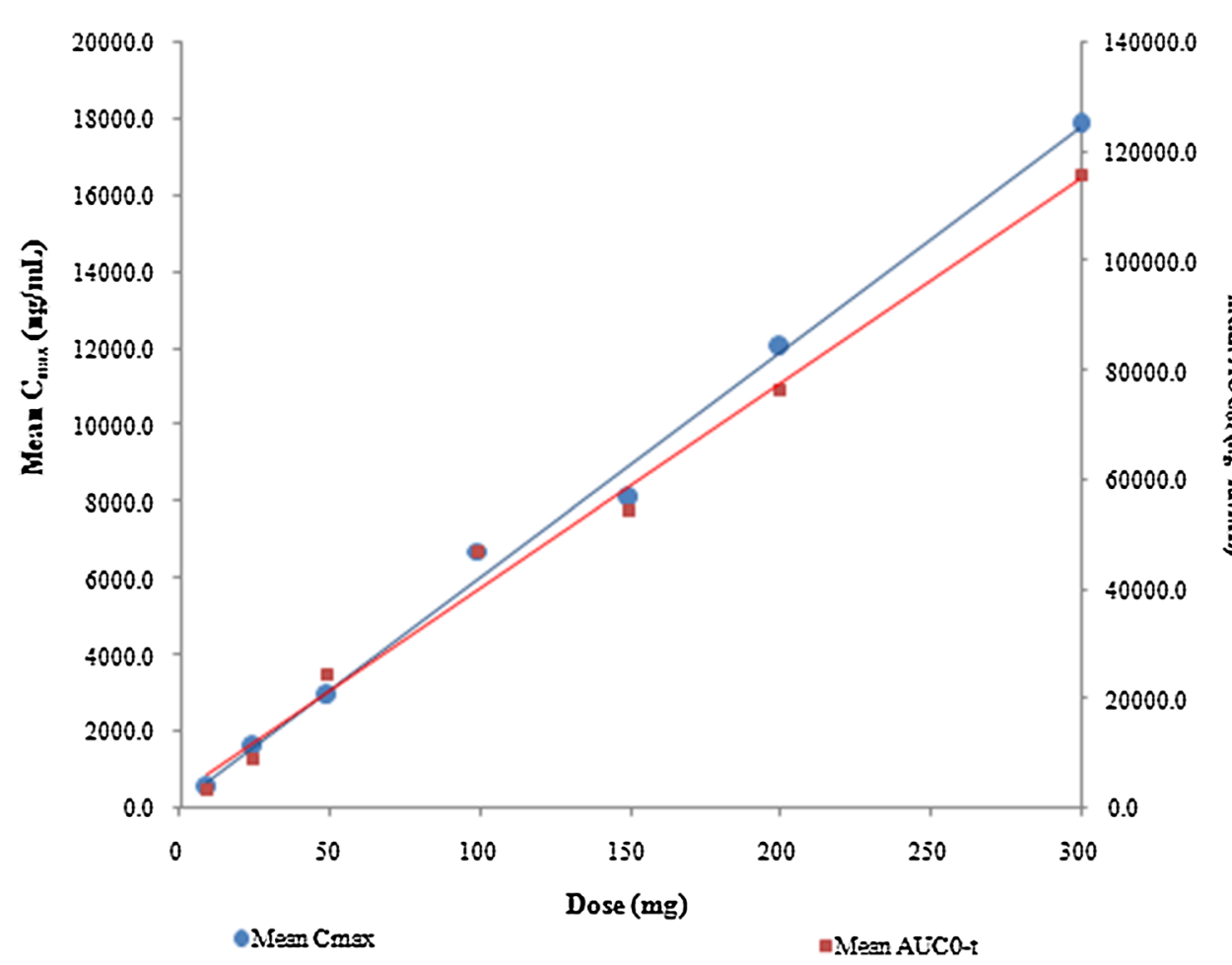

6.9-11.3 h) was dose independent and the parallel decay in the log terminal phases of various doses is evident in Fig. 2. The somewhat shorter $t_{1 / 2}$ values observed at the lowest dose levels (i.e., 10 and $25 \mathrm{mg}$ ) was attributable to sample collection timepoints in the terminal phase of elimination of ZYAN1 (instead of $48 \mathrm{~h}$, samples were collected for $72 \mathrm{~h}$ ). After single oral ZYAN1 administration, the amount of ZYAN1 recovered in urine was $3.63 \pm 1.02,9.45 \pm 1.76,18.99 \pm 3.36,41.13 \pm 12.06$, $56.23 \pm 17.26,62.83 \pm 18.11$, and $82.66 \pm 39.25 \mathrm{mg}$ for the $10,25,50,100,150,200$, and $300 \mathrm{mg}$ dose of ZYAN1, respectively. It was observed that percentage of ZYAN1 recovered in urine ranged from 27 to $42 \%$ after a single dose from 10 to $300 \mathrm{mg}$ (Table 2). The apparent oral CL (range $2-2.9 \mathrm{~L} / \mathrm{h}$ ) and $V_{\mathrm{d}}$ (range $28-47 \mathrm{~L}$ ) parameters showed remarkable consistency across the single doses of ZYAN1 administered in the study.

\subsubsection{Multiple Ascending-Dose Study}

The multiple-dose (Day 1, 3, and 5 dosing) mean concentration versus time profiles of ZYAN1 for the four doses used in the study are presented in Fig. 4. Based on the similarity of the plasma profiles of ZYAN1, the data unambiguously supported lack of accumulation after alternate-day dosing in any of the dosing cohorts. Additionally, if common timepoints such as pre-dose, $12 \mathrm{~h}$, or $24 \mathrm{~h}$ post-dose are examined, there appears to be consistency in the concentrations of ZYAN1. The mean pharmacokinetic data after multiple ascending doses of ZYAN1 are provided in Table 3. Almost identical mean $C_{\max }$ values were observed at the first and fifth day of dosing for the 150, 200, and $300 \mathrm{mg}$ doses of ZYAN1 (Fig. 4). The median $t_{\max }$ of ZYAN1 was observed at around 1.5-3.0 $\mathrm{h}$ after administration of the 100, 150, 200, and $300 \mathrm{mg}$ doses either on the first day or the fifth day of dosing, suggesting that similar absorption rates occur regardless of the dose size or the day of dosing. The mean $t_{1 / 2}$ of ZYAN1 after multiple doses was observed to be between 9.4 and $13 \mathrm{~h}$ for ZYAN1 after the last day of dosing. Furthermore, the percentage recovered in urine of ZYAN1 for the $100,150,200$, and $300 \mathrm{mg}$ dose ranged from 30 to $50 \%$ (Table 4). Based on the mean terminal $t_{1 / 2}$ value of approximately $10 \mathrm{~h}$, it was expected that ZYAN1 was unlikely to show accumulation when orally administered on alternate days. Accordingly, no accumulation of the drug was observed after the first and third dose administration of ZYAN1 100, 150, 200, and $300 \mathrm{mg}$ (Table 3).

\subsubsection{Sex and Food Effect Study}

The mean pharmacokinetic data for fasted and fed subjects are provided in Table 5.

The $t_{\max }$ was delayed under fed conditions with a median $t_{\max }$ value of $5 \mathrm{~h}$ compared with $2 \mathrm{~h}$ under fasting conditions. In addition, the ingestion of food decreased the $C_{\max }$ by 2.39 times as compared with the data obtained 


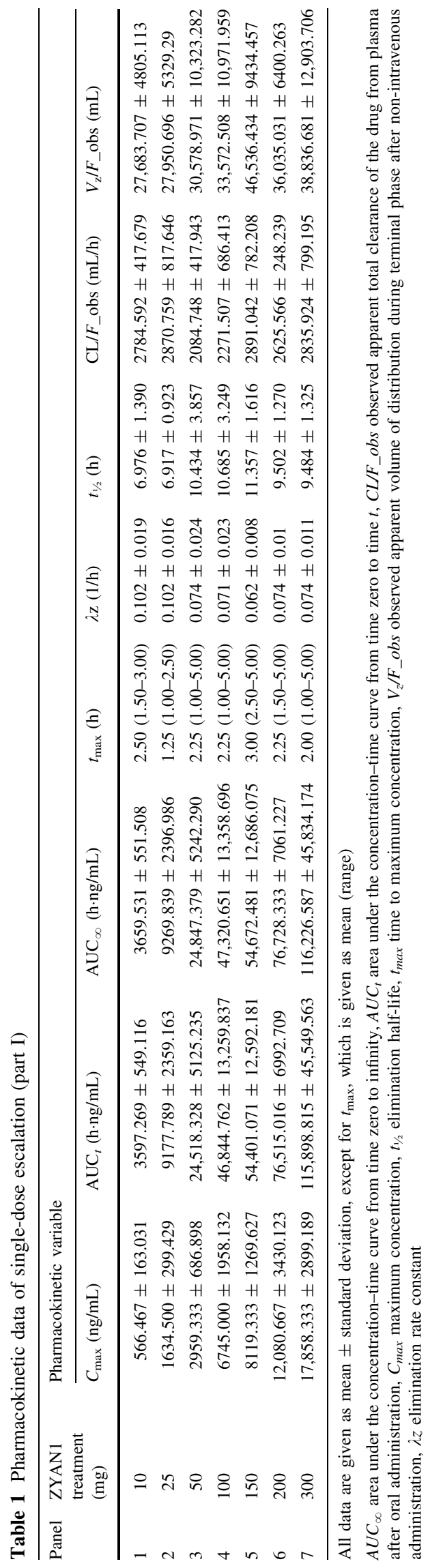

under fasting conditions. The AUC values were decreased in fed conditions as compared with the fasting conditions after administration of ZYAN1. The mean $t_{1 / 2}$ of ZYAN1 was similar between fed vs. fasted treatments. The mean $C_{\max }$ for male and female subjects was not statistically significant but the extent of absorption appeared to be affected by sex.

\subsection{Pharmacodynamic Results}

This study was conducted in healthy volunteers with a normal hemoglobin range, and given the nature of the pharmacokinetic procedures there was blood loss during the study. Therefore, pharmacodynamic results that were solely based on key blood parameters may have to be interpreted with caution.

\subsubsection{Single Ascending-Dose Study}

In a single-dose panel at each dose level, changes in hemoglobin, hematocrit, reticulocyte count, and EPO were observed. A hemoglobin increase of $>0.5 \mathrm{gm} \%$ was observed in most of the subjects receiving ZYAN1 tablets and the increase appeared to be more consistent starting from the oral dose of ZYAN1 $100 \mathrm{mg}$ and above. There was a dose response for mean serum EPO levels for doses of ZYAN1 from $100 \mathrm{mg}$ up to $300 \mathrm{mg}$, which represented an almost proportional EPO increase with increasing ZYAN1 doses. The mean serum EPO $C_{\max }$ values versus time curve showed peak levels of EPO ranging from $6.6 \mathrm{mIU} / \mathrm{L}$ for ZYAN1 $10 \mathrm{mg}$ to $79.9 \mathrm{mIU} / \mathrm{L}$ for ZYAN1 $300 \mathrm{mg}$ (Fig. 5), while in subjects receiving placebo, the $C_{\max }$ of EPO observed was $9.6 \mathrm{mIU} / \mathrm{L}$. The mean serum EPO $t_{\max }$ ranged from 10 to $72 \mathrm{~h}$, with mean serum EPO returning to baseline levels within $24 \mathrm{~h}$.

There was no apparent change in mean hematocrit, hemoglobin and reticulocyte counts over time or between various treatment doses (ZYAN1 vs. placebo) after single ascending doses of ZYAN1.

\subsubsection{Multiple Ascending-Dose Study}

In the multiple-dose phase of this study (part II), mean serum EPO concentrations increased following each dose of ZYAN1 (on Days 1, 3, and 5), with a dose-related response at ZYAN1 200 and $300 \mathrm{mg}$. We observed that the serum EPO $C_{\max }$ values ranged from $14.4 \mathrm{mIU} / \mathrm{L}$ for ZYAN1 $100 \mathrm{mg}$ to $60.5 \mathrm{mIU} / \mathrm{L}$ for ZYAN1 $300 \mathrm{mg}$ while it was $11.0 \mathrm{mIU} / \mathrm{L}$ for subjects who received placebo. The serum EPO profile after multiple dosing followed a similar profile to single dosing, with a lag in time of approximately 6-8 h to EPO $C_{\max }$ from pharmacokinetic $t_{\max }$ for ZYAN1 (Fig. 6). 
Table 2 Descriptive statistics for urine data for part I

\begin{tabular}{llcl}
\hline ZYAN1 treatment $(\mathrm{mg})$ & Panel & $\begin{array}{l}\text { Amount recovered }(\mathrm{mg}) \\
{[\text { mean } \pm \mathrm{SD}]}\end{array}$ & $\begin{array}{l}\text { Percentage recovered (\%) } \\
{[\text { mean } \pm \text { SD] }}\end{array}$ \\
\hline 10 & 1 & $3.63 \pm 1.02$ & $36.270 \pm 10.245$ \\
25 & 2 & $9.45 \pm 1.76$ & $37.798 \pm 7.059$ \\
50 & 3 & $18.99 \pm 3.36$ & $37.995 \pm 6.718$ \\
100 & 4 & $41.13 \pm 12.06$ & $41.130 \pm 12.064$ \\
150 & 5 & $56.23 \pm 17.26$ & $37.485 \pm 11.508$ \\
200 & 6 & $62.83 \pm 18.11$ & $31.417 \pm 9.056$ \\
300 & 7 & $82.66 \pm 39.25$ & $27.555 \pm 13.083$ \\
\hline
\end{tabular}

$S D$ standard deviation

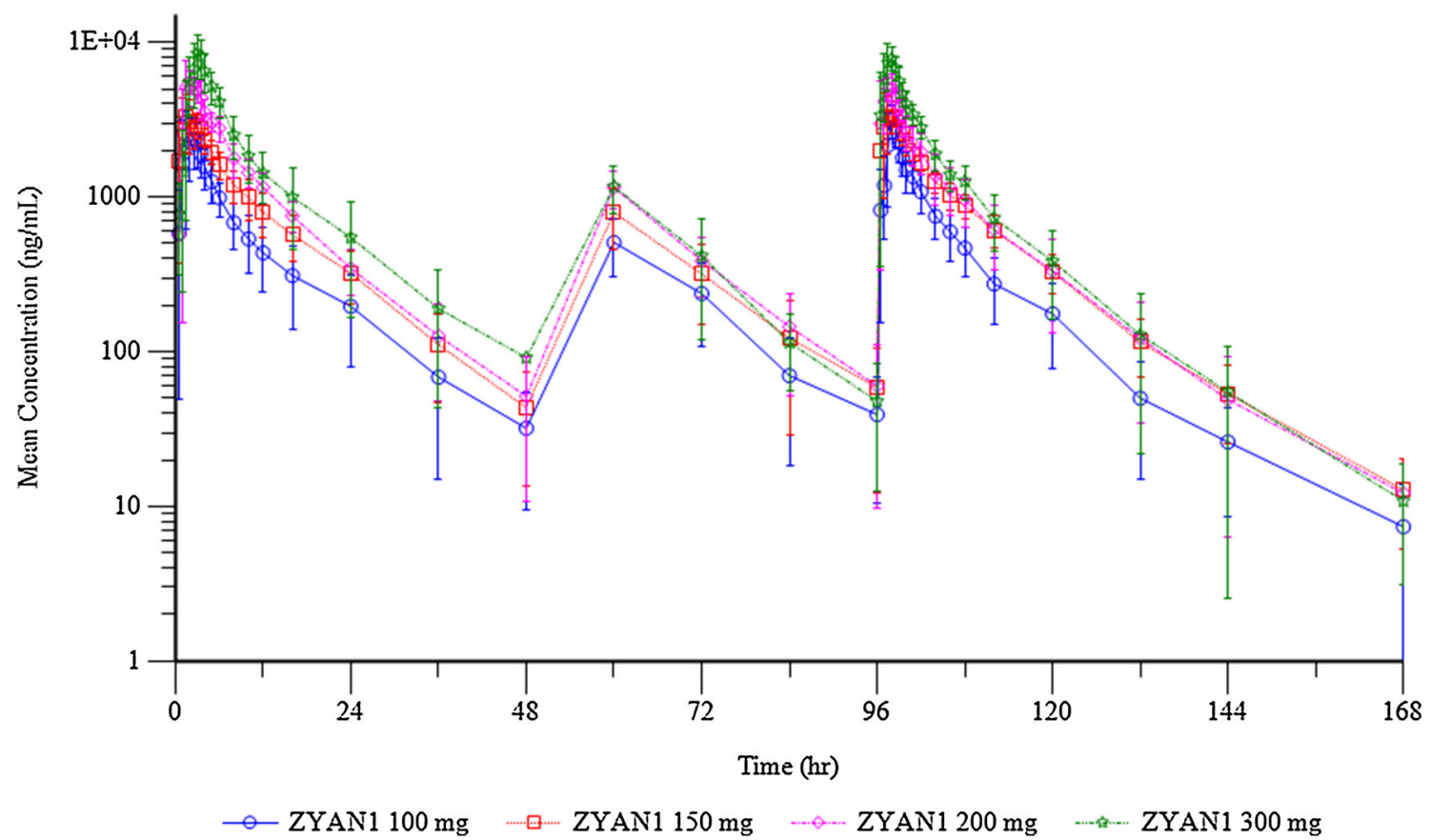

Fig. 4 Mean concentration vs. time graph with error bars for part II

It was observed that a higher number of subjects who received ZYAN1 showed a hemoglobin increase of $>0.5$ gm\% on any timepoint than in the placebo group. A hemoglobin increase $>1.0 \mathrm{gm} \%$ on any timepoint was also seen in more subjects who received ZYAN1 than in those who received placebo. There was no apparent change in mean hematocrit, hemoglobin, and reticulocyte count over time or between treatments (ZYAN1 vs. placebo) and there was no apparent dose response following ZYAN1 administration.

\subsubsection{Sex and Food Effect Study}

There was no apparent difference in mean serum EPO concentrations in subjects under fasted conditions compared with fed conditions (Fig. 7). In addition, there was no apparent difference in mean serum EPO concentrations between male and female subjects under either fasted or fed conditions. Similarly, there were no apparent differences in mean hematocrit, hemoglobin, and reticulocyte count over time under fasted conditions compared with fed conditions, and no apparent difference between male and female subjects under either fasted or fed conditions.

\subsection{Safety Evaluation}

\subsubsection{Single Ascending-Dose Study}

A total of 20 AEs were reported in the single ascendingdose study. Of the AEs deemed possibly (?) related to study treatment, nine were reported in six subjects $(14 \%)$ who received ZYAN1 and three were reported in three subjects (21\%) who received placebo, while the rest of the AEs were not related to investigational product. All AEs deemed to be possibly related to study treatment (?) were grade 1 in severity. The reported AEs, mostly one episode 


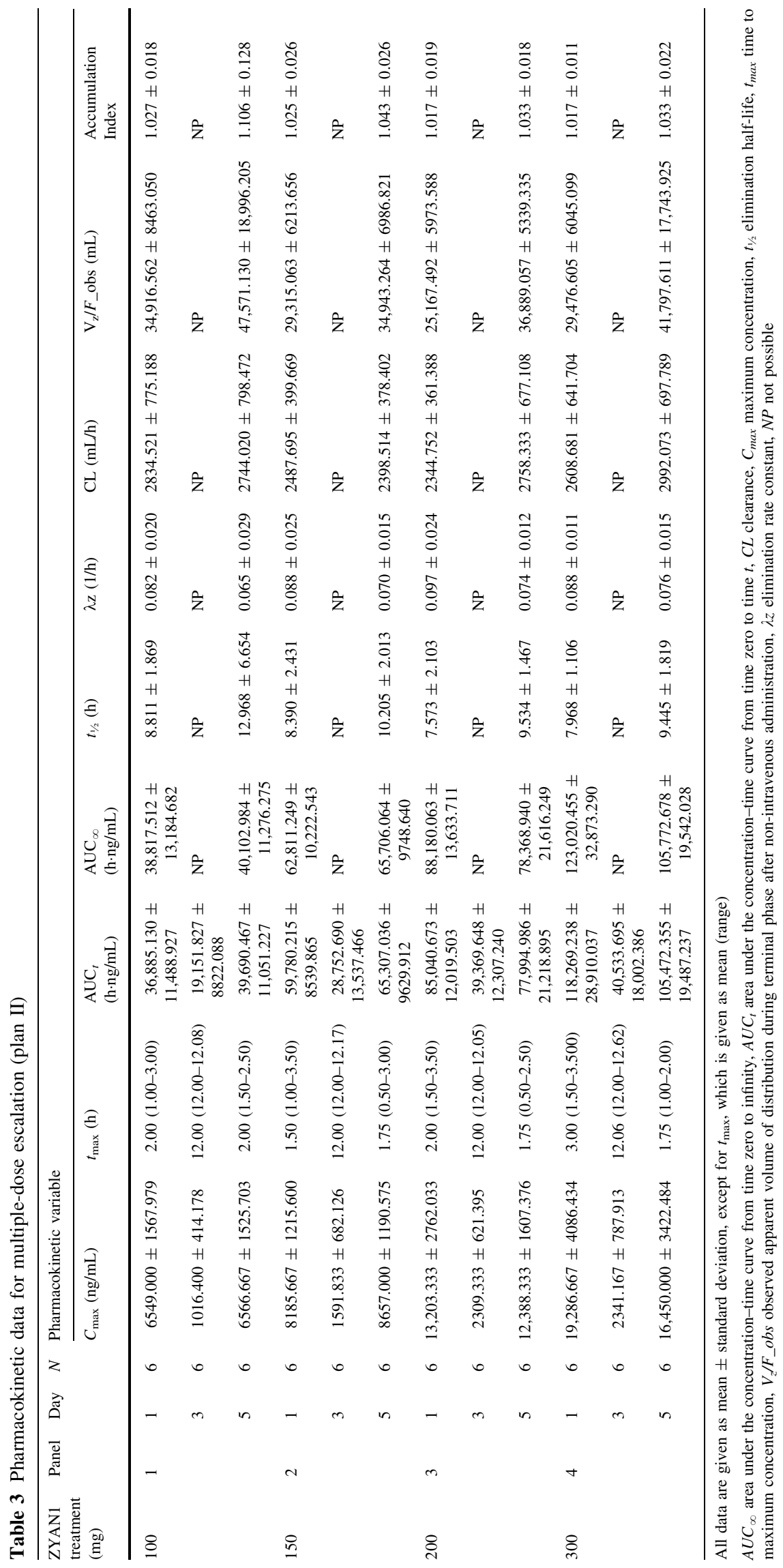


Table 4 Descriptive statistics for urine data for part II

\begin{tabular}{|c|c|c|c|c|}
\hline $\begin{array}{l}\text { ZYAN1 treatment } \\
(\mathrm{mg})\end{array}$ & Panel & Day & $\begin{array}{l}\text { Amount recovered } \\
(\mathrm{mg})[\text { mean } \pm \mathrm{SD}]\end{array}$ & $\begin{array}{l}\text { Percentage recovered } \\
(\%)[\text { mean } \pm \mathrm{SD}]\end{array}$ \\
\hline \multirow[t]{3}{*}{100} & \multirow[t]{3}{*}{1} & 1 & $50.13 \pm 14.99$ & $50.132 \pm 14.998$ \\
\hline & & 3 & $41.00 \pm 11.07$ & $41.001 \pm 11.071$ \\
\hline & & 5 & $50.14 \pm 12.16$ & $50.137 \pm 12.160$ \\
\hline \multirow[t]{3}{*}{150} & \multirow[t]{3}{*}{2} & 1 & $52.77 \pm 6.85$ & $35.178 \pm 4.566$ \\
\hline & & 3 & $54.64 \pm 13.35$ & $36.427 \pm 8.900$ \\
\hline & & 5 & $44.23 \pm 8.18$ & $29.488 \pm 5.453$ \\
\hline \multirow[t]{3}{*}{200} & \multirow[t]{3}{*}{3} & 1 & $64.95 \pm 25.50$ & $32.474 \pm 12.749$ \\
\hline & & 3 & $66.09 \pm 8.74$ & $33.044 \pm 4.371$ \\
\hline & & 5 & $75.76 \pm 12.76$ & $37.880 \pm 6.381$ \\
\hline \multirow[t]{3}{*}{300} & \multirow[t]{3}{*}{4} & 1 & $82.97 \pm 35.69$ & $27.656 \pm 11.899$ \\
\hline & & 3 & $92.22 \pm 41.92$ & $30.741 \pm 13.972$ \\
\hline & & 5 & $99.15 \pm 34.40$ & $33.050 \pm 11.466$ \\
\hline
\end{tabular}

$S D$ standard deviation

\begin{tabular}{lll}
\hline Variable & \multicolumn{2}{l}{ Condition $(n=10)$} \\
\cline { 2 - 3 } & Fasting & Fed \\
\hline$C_{\max }(\mathrm{ng} / \mathrm{mL})$ & $10,352.800 \pm 3076.528$ & $4322.000 \pm 1317.961$ \\
$\mathrm{AUC}_{t}(\mathrm{~h} \cdot \mathrm{ng} / \mathrm{mL})$ & $71,331.577 \pm 25,663.234$ & $59,005.375 \pm 16,846.249$ \\
$\mathrm{AUC}_{\infty}(\mathrm{h} \cdot \mathrm{ng} / \mathrm{mL})$ & $71,696.758 \pm 25,837.440$ & $59,561.279 \pm 17,228.977$ \\
$t_{\text {max }}(\mathrm{h})$ & $2.000(1.000-3.500)$ & $5.000(1.000-12.000)$ \\
$\lambda \mathrm{z}(1 / \mathrm{h})$ & $0.067 \pm 0.015$ & $0.066 \pm 0.017$ \\
$\mathrm{HL} \_$Lambda_z $(\mathrm{h})$ & $11.048 \pm 3.378$ & $11.328 \pm 3.691$ \\
$\mathrm{CL} / F_{-}$obs $(\mathrm{mL} / \mathrm{h})$ & $2479.865 \pm 1333.719$ & $2801.105 \pm 1109.706$ \\
$V_{\mathrm{z}} / F_{-}$obs $(\mathrm{mL})$ & $37,927.046 \pm 18,082.141$ & $45,070.176 \pm 19,928.748$
\end{tabular}

All data are given as mean \pm standard deviation, except for $t_{\max }$, which is given as mean (range)

$A U C_{\infty}$ area under the concentration-time curve from time zero to infinity, $A U C_{t}$ area under the concentration-time curve from time zero to time $t, C L / F_{\_}$obs observed apparent total clearance of the drug from plasma after oral administration, $C_{\max }$ maximum concentration, $t_{\max }$ time to maximum concentration, $V_{z} /$ $F \_o b s$ observed apparent volume of distribution during terminal phase after non-intravenous administration, $\lambda z$ elimination rate constant, $H L \_L a m b d a \_z$ terminal half-life each, were rhinorrhea, tooth pain, sore throat, dizziness, headache, chest pain, diarrhea, upper respiratory tract infection, lethargy, nausea, throat irritation, increase in aspartate aminotransferase (AST), hyperkalemia, and back pain. No AEs were classified as grade 3 .

\subsubsection{Multiple Ascending-Dose Study}

A total of 32 AEs were reported in the multiple ascendingdose study. Of the AEs possibly related to study treatment, 16 were reported in 11 subjects $(46 \%)$ who received ZYAN1 and eight were reported in five subjects $(63 \%)$ who received placebo. These treatment-related AEs were all grade 1 in severity, with the exception of dizziness in two subjects, which was classified as grade 2 in severity. The reported AEs were headache, dizziness, upper respiratory tract infection, nausea, diarrhea, phlebitis, sinus tachycardia, dyspepsia, supra ventricular extrasystoles, abdominal pain, contusion, back pain, and nasal congestion. No AEs were classified as grade 3 .

\subsubsection{Sex and Food Effect Study}

A total of 11 AEs were reported in this crossover study at a dose of ZYAN1 $150 \mathrm{mg}$. One subject reported a SAE (grade 3) of cellulitis after 2 days of dosing; the subject was withdrawn from the study. The SAE was deemed not to be related to the study drug and resolved sequentially. Overall, in part III of the study, six AEs were classified as grade 1 in severity, four were classified as grade 2 in severity (headache [three AEs, one possibly related, two not related] and musculoskeletal pain [one AE, not related]), and one AE was classified as grade 3 (cellulitis, not related). 


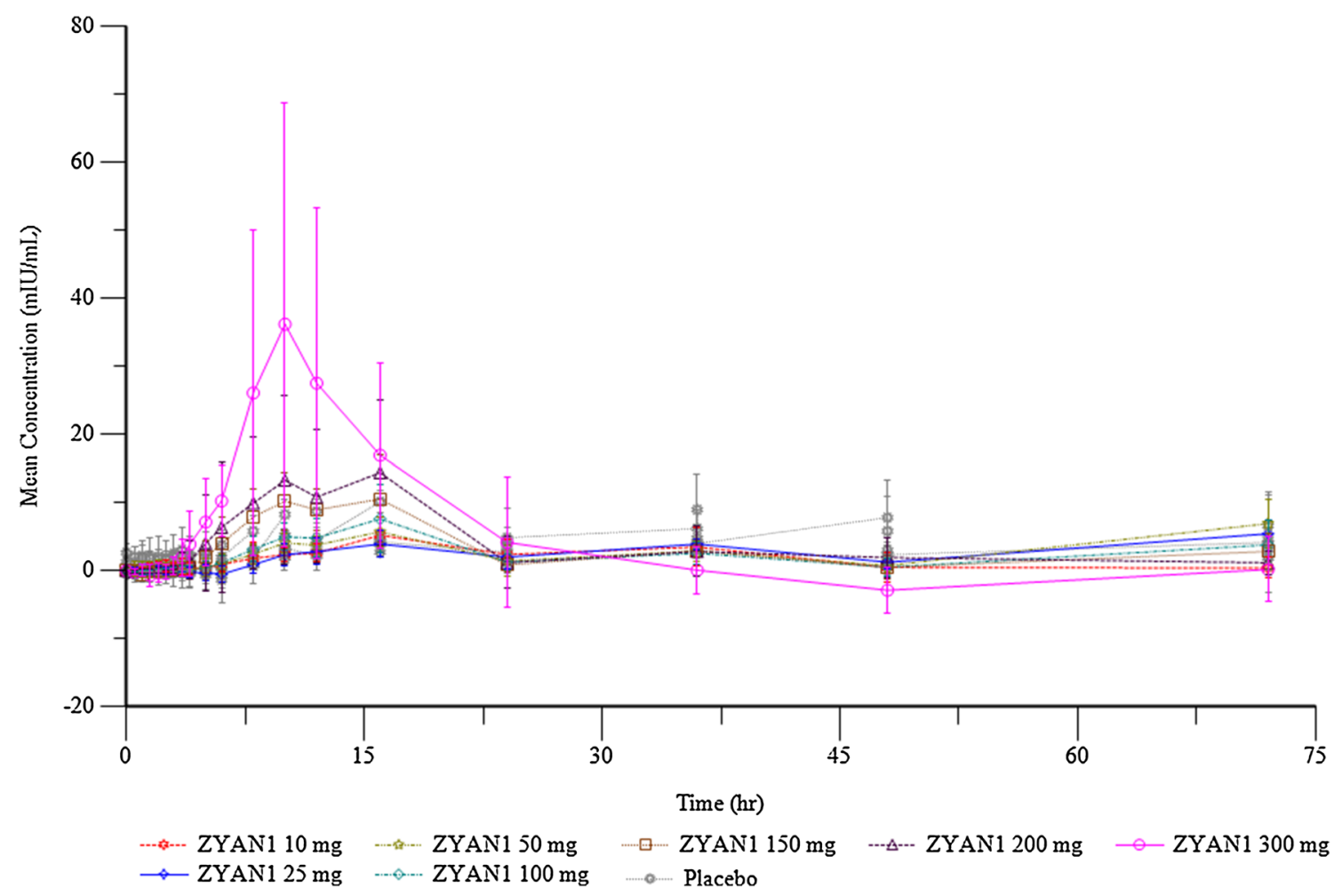

Fig. 5 Mean concentration of erythropoietin for part I (baseline corrected)

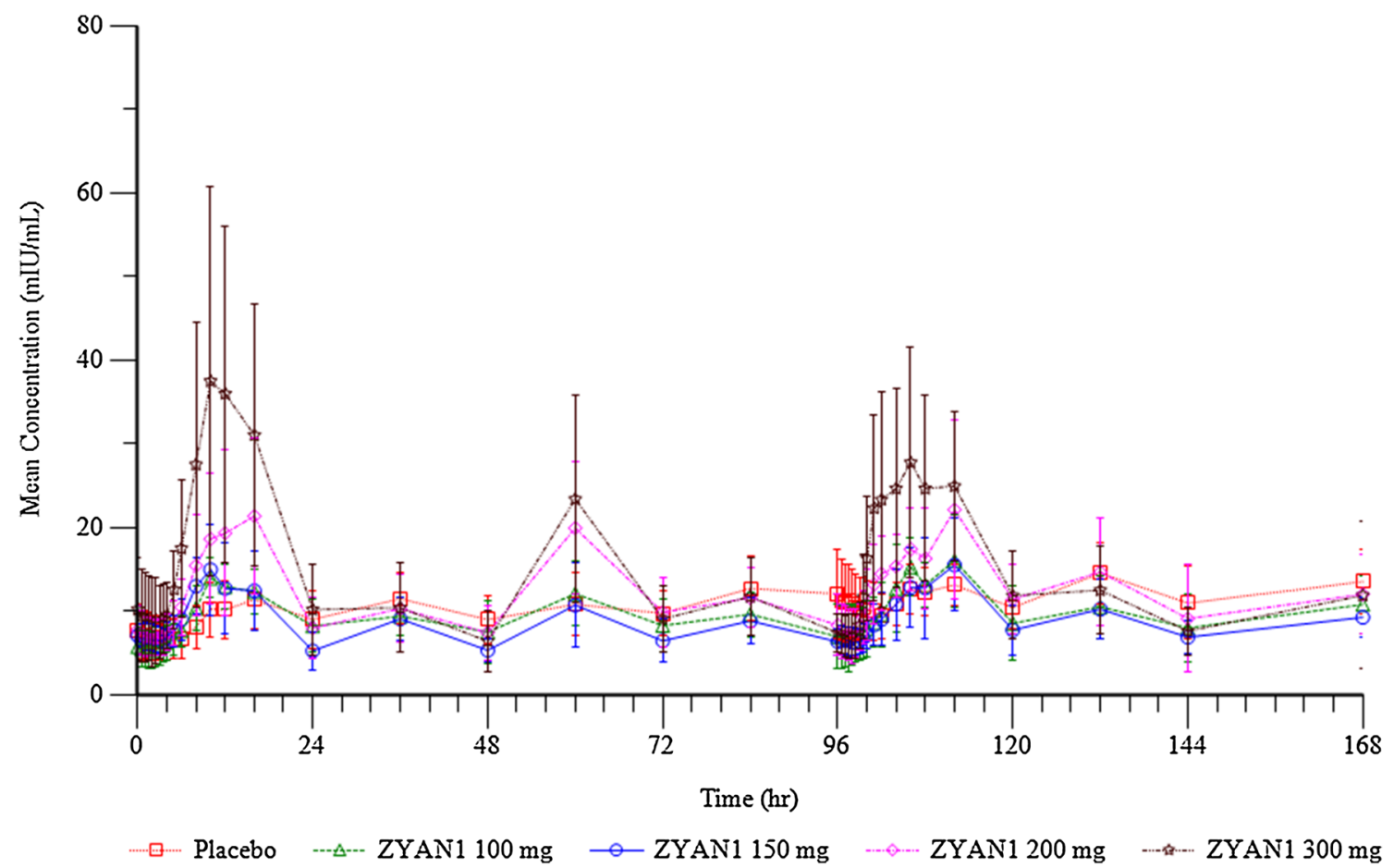

Fig. 6 Mean concentration of erythropoietin for part II (baseline corrected) 


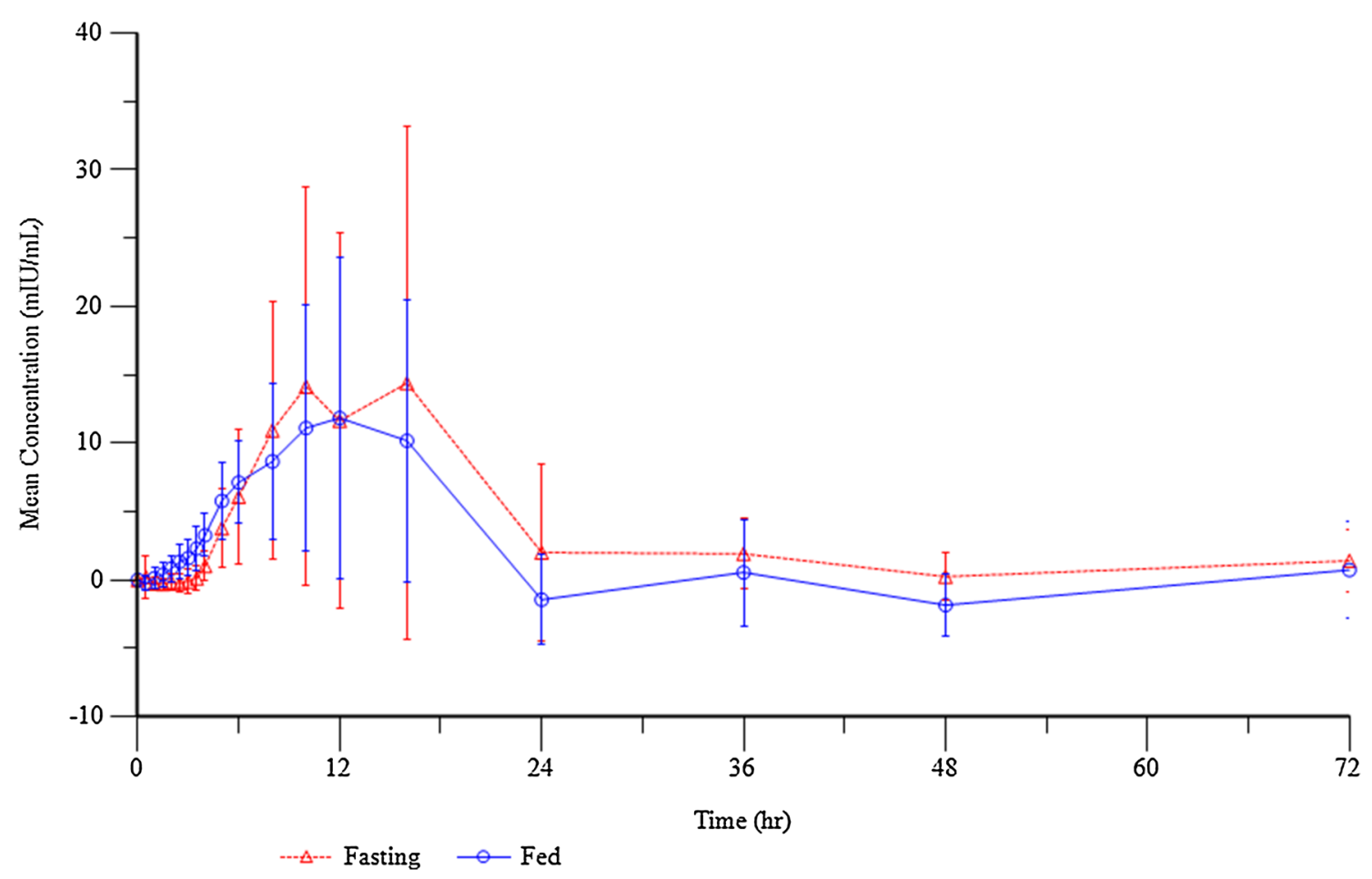

Fig. 7 Mean concentration of erythropoietin for part III (baseline corrected)

In addition, among the three studies, general safety was indistinguishable between active dose (ZYAN1) and placebo subjects, as assessed by clinical laboratory tests, vital signs, ECG assessments, and cardiac telemetry in the single ascending-dose, multiple ascending-dose, and food effect studies. The AEs observed in the study are summarized in Table 6.

\section{Discussion}

There has been considerable interest in PHD inhibitors for the treatment of anemia, with a few compounds, namely roxadustat (FG-4592), molidustat (BAY85-3934) and vadadustat, already being evaluated in late-phase clinical trials [26]. Therefore, we were interested in evaluating the safety, tolerability, pharmacokinetics, and pharmacodynamics of ZYAN1, a potent orally administered HIF-PHD inhibitor, to develop a comprehensive phase I package to aid in the planning of phase II development of this drug.

In totality, this phase I evaluation comprised 100 healthy volunteers, assigned to single ascending-dose panels, multiple ascending-dose panels, and a food/sex effect panel. In order to avoid the potential risk of an overt drug exposure in a larger number of subjects, each dose panel of eight subjects was subdivided into two blocks (applicable for parts 1 and II). In the first block, two subjects were enrolled (one placebo + one ZYAN1), while in the second block, six subjects were enrolled (one placebo + five
ZYAN1). The dosing of the second block of each panel was initiated $24 \mathrm{~h}$ post-dose and when the safety analysis of the first block was available.

Due to the nature of the study design, the pharmacokinetics of ZYAN1 were assessed under various conditions with extensive blood sampling to provide guidance for further development of the drug. The pharmacodynamics of ZYAN1 were monitored based on the assessment of hemoglobin, hematocrit, reticulocyte count, and EPO level. For safety assessment, $\mathrm{AE}$ and SAE reports, clinical examination, vitals, ECG, and clinical laboratory assessment were considered.

The single ascending-dose study of ZYAN1 suggested an almost dose-proportional increase in the exposure (i.e., $C_{\max }$ and AUC) in relation to a 30 -fold range of orally administered ZYAN1. The absorption of ZYAN1 appeared to be relatively rapid and consistent among the various single doses. As the ZYAN1 doses increased, the $V_{\mathrm{d}}$ remained relatively consistent, suggesting a lack of non-linear distribution of the drug at higher doses. The apparent CL of ZYAN1 was similar across the dose levels, suggesting a lack of saturation in the elimination and/or excretory process of ZYAN1. Due to comparable CL and $V_{\mathrm{d}}$, the $t_{1 / 2}$ did not vary as a function of dose.

The multiple ascending-dose pharmacokinetics of ZYAN1 mimicked the single-dose data with no accumulation of drug as judged by $C_{\max }$ and AUC values between Day 1 and Day 5 dosing of the drug. In addition, the $t_{1 / 2}$ 
Table 6 Summary of adverse events

\begin{tabular}{lll}
\hline $\begin{array}{l}\text { ZYAN1 Panel } \\
\text { mg })\end{array}$ & Adverse events & Placebo $(n=22)$ \\
\cline { 2 - 3 } & Test $(n=78)$ & Nil \\
\hline SAD 10 & Nil & Nil \\
SAD 25 & Rhinorrhea, right lower wisdom tooth pain, sore throat & Nil \\
SAD 50 & Dizziness, headache, chest pain, Diarrhea, upper respiratory tract infection & Dizziness, headache \\
SAD 100 & Dizziness, headache, lethargy, nausea, throat irritation, increase in AST, hyperkalemia & Nil \\
SAD 150 & Nil & Nil \\
SAD 200 & Back pain & Headache \\
SAD 300 & Nil & Upper respiratory tract \\
MSD 100 & Headache, dizziness & Headache, dizziness \\
MAD 150 & Headache, dizziness & Sinus tachycardia, \\
MAD 200 & Nausea, headache, dizziness, diarrhea, phlebitis & dyspepsia, dizziness \\
MAD 300 & Supra ventricular extrasystoles, abdominal pain, diarrhea, contusion, back pain, dizziness, & Nil \\
Food and sex & Palpitation, cellulitis, back pain, musculoskeletal pain, head discomfort, headache (2), & Headache \\
effect 150 & folliculitis, cellulitis &
\end{tabular}

$A S T$ aspartate aminotransferase, $M A D$ multiple ascending-dose, $S A D$ single ascending-dose

values after multiple doses were found to be in a similar range to that observed for the single dose. It was also observed that the amount and percentage recovered in urine was comparable after every dose of ZYAN1 in the multiple-dose study and also similar to the observations seen with the respective single dose of ZYAN1.

The exploratory food effect study showed that both the rate and extent of absorption of ZYAN1 was altered by concomitant food intake. Similarly, the analysis between sexes suggested that female subjects had a higher exposure than males. However, as it was a small subject group, the data represents qualitative difference at best, but definitive conclusions cannot be inferred.

Our results showed that following a single dose of ZYAN1, mean serum EPO $C_{\max }$ values increased over time, with peak levels ranging from $6.6 \mathrm{mIU} / \mathrm{L}$ for ZYAN1 $10 \mathrm{mg}$ to $79.9 \mathrm{mIU} / \mathrm{L}$ for ZYAN1 $300 \mathrm{mg}$. The initiation of EPO rise was observed as early as after $6 \mathrm{~h}$ of ZYAN1 administration and an increase of almost 2- to 4-fold was observed compared with baseline in the 150, 200, and $300 \mathrm{mg}$ panels. The EPO response was not markedly different from placebo for doses up to ZYAN1 $150 \mathrm{mg}$. There was no apparent difference observed for EPO between males and females or between fasted and fed conditions. With a single dose of ZYAN1, the effect on the biomarker to support the mechanism of action of our compound was observed [27, 28].

We observed a hemoglobin increase of $>0.5 \mathrm{gm} \%$ in most of the subjects after a ZYAN1 single dose, with a more consistent rise with $100 \mathrm{mg}$ and higher dosing.
Similar hemoglobin response was seen in multiple-dose panels even though the subjects had also undergone some study-related blood loss. This may suggest the ability of ZYAN1 to participate in erythropoiesis.

There was no apparent change in mean hematocrit, hemoglobin, and reticulocyte count over time or between treatments (ZYAN1 vs. placebo), and there was no apparent dose response following ZYAN1 administration.

In parts I, II, and III of the study, there were no clear differences in safety as assessed by clinical laboratory tests, vital signs, ECG assessments, and cardiac telemetry in subjects who had received either single or multiple doses of ZYAN1 compared with those who received placebo, and no dose-related trends in these safety assessments in subjects who received ZYAN1.

In parts I and II of the study, there were no apparent dose-related trends for any of the commonly occurring AEs. Common AEs of headache and dizziness were reported in both part I (single dose) and part II (multiple doses) of the study, the majority of which were deemed related to the study drug (including subjects who received placebo). Moreover, these commonly occurring AEs are similar to those seen with other PHD inhibitor compounds and no new AEs were reported [26].

While the urinary excretion of intact ZYAN1 was confirmed in healthy subjects (average 36\%), supporting the importance of renal function, it also suggested the role of non-renal CL mechanisms for the elimination of ZYAN1. In this context, the pharmacokinetics of vadadustat were not found to be significantly altered in CKD patients 
(stages 3 and 4) despite the role of renal CL mechanisms (approximately 59\% of the radioactive dose) in the disposition of 14C-vadadustat in healthy subjects [29]. To underscore this observation, we have found that both acute kidney failure and chronic kidney failure models in rats did not alter the pharmacokinetics of ZYAN1 as compared with those in normal controls rats [30]. It was also noted that complete removal of both kidneys in rats led to a 2-fold increase in the exposure of ZYAN1, suggesting the role of non-renal CL pathways for the elimination of ZYAN1 [30].

The study was conducted in healthy volunteers mainly to evaluate safety, tolerability, pharmacokinetics, and pharmacodynamics. Therefore, safety and efficacy data derived from this study should be viewed with caution while also considering the patient group. Moreover, the study involved blood loss in the form of collection of blood samples for the pharmacokinetics, pharmacodynamics, and safety assessment, which perhaps had some influence with the efficacy parameters evaluated in the study.

As discussed in Sect. 1, hepcidin, a key regulator of iron absorption and remobilization, is normally down-regulated by erythropoiesis, anemia, and hypoxia. HIF stabilization, via the PHD inhibitor, is associated with hepcidin suppression, which increases intestinal iron absorption and also increases enzymes of iron transportation [26]. As current therapeutic options for the management of anemia of CKD have certain limitations, development of HIF-PHD inhibitors to favorably modify the HIF-PHD pathway can be helpful for the management of anemia with CKD patients. However, based on the observed results of this study, ZYAN1 is a safe and effective ESA for the treatment of ACDs. The proposed dose range of 100-200 mg every 2 days appears to be reasonable for the phase II development of ZYAN1 in this patient population.

\section{Conclusion}

Overall, ZYAN1 was well-tolerated when administered as single oral doses (or multiple oral doses with alternate-day dosing) in healthy male subjects. Serum EPO levels showed dose response in the ZYAN1 oral doses evaluated. Based on the $t_{1 / 2}$, pharmacodynamic activity, and lack of drug accumulation, an every 2 days dosing regimen of ZYAN1 is appropriate for phase II study. The proposed dose range of ZYAN1 for phase II study is 100-200 mg every 2 days.

\section{Compliance with Ethical Standards}

Funding Dr Jason Lickliter, Centre for Clinical Studies (CCS), a division of Nucleus Network, Alfred Medical Research and CPR Pharma Services Pty. Ltd, Australia have received funding from
Cadila Healthcare Ltd. Dr. Deven Parmar, an employee of Cadila Healthcare Ltd., provided assistance with writing, formatting, proof reading, and collation of the author comments. Open access publication of this study was sponsored by Cadila Healthcare Ltd. Cliantha Research Limited and Karmic Life Sciences have received funding from Cadila Healthcare Ltd. for statistical analysis and data management, respectively.

Conflict of interest Kevinkumar A. Kansagra, Rajendra H. Jani, Nuggehally R. Srinivas, Jason Lickliter, Harilal V. Patel, Devang P. Parikh, Heather Heading, Hardik B. Patel, Rahul J. Gupta, Chintan Y. Shah, Maulik R. Patel, Vyom N. Dholakia, Raghav Sukhadiya, Mukul R. Jain, Krupi V. Parmar, and Kinjal Barot have no conflicts of interest related to this study to report.

Ethical approval All of the study-related procedures were performed after gaining approval from the Ethics Committee of the Centre for Clinical Studies and in accordance with the ethical standards set forth by them and with the 1964 Helsinki declaration and its later amendments or comparable ethical standards.

Informed consent Written informed consent was obtained from all individual participants before enrollment into the study.

Open Access This article is distributed under the terms of the Creative Commons Attribution-NonCommercial 4.0 International License (http://creativecommons.org/licenses/by-nc/4.0/), which permits any noncommercial use, distribution, and reproduction in any medium, provided you give appropriate credit to the original author(s) and the source, provide a link to the Creative Commons license, and indicate if changes were made.

\section{References}

1. Spinowitz BS, Kausz AT, Baptista J, Noble SD, Sothinathan R, Bernardo MV, et al. Ferumoxytol for treating iron deficiency anemia in CKD. J Am Soc Nephrol. 2008;19:1599-605.

2. Agarwal N, Prchal JT. Anemia of chronic disease (anemia of inflammation). Acta Haematol. 2009;122(2-3):103-8.

3. Hayes JM. Chemotherapy induced anemia: treating it as a disease not just as a condition. Adv Stud Pharm. 2007;5(5):125-32.

4. Haase VH. Regulation of erythropoiesis by hypoxia-inducible factors. Blood Rev. 2013;27(1):41-53.

5. Wang J, Pantopoulos K. Regulation of cellular iron metabolism. Biochem J. 2011;434(Pt 3):365-81.

6. Nemeth E, Genz T. Anemia of inflammation. Hematol Oncol Clin N Am. 2014;28(4):671-81.

7. Ganz T, Nemeth E. Hepcidin and iron homeostasis. Biochim Biophys Acta. 2012;1823(9):1434-43.

8. Karuppagounder SS, Ratan RR. Hypoxia-inducible factor prolyl hydroxylase inhibition: robust new target or another big bust for stroke therapeutics? J Cereb Blood Flow Metab. 2012;32(7):1347-61.

9. Maxwell PH, Eckardt K. HIF prolyl hydroxylase inhibitors for the treatment of renal anaemia and beyond. Nat Rev Nephrol. 2016;12:157-68.

10. Jain MR, Joharapurkar AA, Pandya V, Patel V, Joshi J, Kshirsagar S, et al. Pharmacological characterization of ZYAN1, a novel prolyl hydroxylase inhibitor for the treatment of anemia. Drug Res. 2016;66(2):107-12.

11. Tsagalis G. Renal anemia: a nephrologist's view. Hippokratia. 2011;15(Suppl 1):39-43.

12. Haase VH. Hypoxic regulation of erythropoiesis and iron metabolism. Am J Physiol Renal Physiol. 2010;299(1):F1-13. 
13. Melnikova I. Anaemia therapies. Nat Rev Drug Discov. 2006;5(8):627-8.

14. Balarajan Y, Ramakrishnan U, Ozaltin E, Shankar AH, Subramanian SV. Anaemia in low-income and middle-income countries. Lancet. 2011;378(9809):2123-35.

15. Muchnik E, Kaplan J. HIF prolyl hydroxylase inhibitors for anemia. Expert Opin Invest Drugs. 2011;20(5):645-56.

16. Rosenberger C, Mandriota S, Jurgensen JS. Expression of hypoxia-inducible factor-1alpha and -2alpha in hypoxic and ischemic rat kidneys. J Am Soc Nephrol. 2002;13(7): 1721-32.

17. Takubo K, Goda N, Yamada W. Regulation of the HIF-1alpha level is essential for hematopoietic stem cells. Cell Stem Cell. 2010;7(3):391-402.

18. Forristal CE, Winkler IG, Nowlan B, Barbier V, Walkinshaw G, Levesque JP. Pharmacologic stabilization of HIF-1alpha increases hematopoietic stem cell quiescence in vivo and accelerates blood recovery after severe irradiation. Blood. 2013;121(5): 759-69.

19. Beuck S, Schaenzer W, Thevis M. Hypoxia-inducible factor stabilizers and other small-molecule erythropoiesis-stimulating agents in current and preventive doping analysis. Drug Test Anal. 2012;4(11):830-45.

20. Bakris GL, Yu KH, Leong R, Shi W, Lee T, Saikali K, et al. Effects of a novel anemia treatment, FG-4592 - an oral hypoxiainducible prolyl hydroxylase inhibitor (HIF-PHI) on blood pressure and cholesterol in patients with chronic kidney disease: LBOR-06. J Clin Hypertens. 2012;14(7):489.

21. Semenza GL, Wang GL. A nuclear factor induced by hypoxia via de novo protein synthesis binds to the human erythropoietin gene enhancer at a site required for transcriptional activation. Mol Cell Biol. 1992;12(12):5447-54.
22. Wang GL, Semenza GL. Purification and characterization of hypoxia-inducible factor 1. J Biol Chem. 1995;270(3):1230-7.

23. Rosen MD, Venkatesan H, Peltier HM, Bembenek SD, Kanelakis $\mathrm{KC}$, et al. Benzimidazole-2-pyrazole HIF prolyl 4-hydroxylase inhibitors as oral erythropoietin secretagogues. ACS Med Chem Lett. 2010;1(9):526-9.

24. Data on file, Cadila Healthcare Ltd., 2014.

25. Guidance for Industry: Bioanalytical Method Validation. US Department of Health and Human Services, Food and Drug Administration, Center for Drug Evaluation and Research and Center for Veterinary Medicine, May 2001.

26. Besarab A, Provenzano R, Hertel J, Zebaneh R, Klaus SJ, Lee T, et al. Randomized placebo-controlled dose-ranging and pharmacodynamics study of roxadustat (FG-4592) to treat anemia in nondialysis-dependent chronic kidney disease patients. Nephrol Dial Transplant. 2015;30(10):1665-73.

27. Bernhardt WM, Wiesener MS, Scigalla P, Chou J, Schmieder RE, Gunzler V, et al. Inhibition of prolyl hydroxylases increases erythropoietin production in ESRD. J Am Soc Nephrol. 2010;21:2151-6.

28. Lee FS, Percy MJ. The HIF pathway and erythrocytosis. Annu Rev Pathol. 2011;6:165e92.

29. Buch A, Maroni B, Hartman C. Dose exposure relationship of vadadustat is independent of the level of renal function [poster no. \#SA-PO537]. In: American Society of Nephrology Kidney Week; 3-8 Nov 2015; San Diego.

30. Patel H, Joharapurkar AA, Pandya VB, Patel VI, Kshirsagar SG, Patel $P$, et al. Influence of acute and chronic kidney failure in rats on the disposition and pharmacokinetics of ZYAN1, a novel prolyl hydroxylase inhibitor, for the treatment of chronic kidney disease-induced anemia. Xenobiotica. 2017;19:1-8. doi:10.1080/ 00498254.2016.1278287. 